\title{
Efecto del flujo secundario en la separación de metales pesados en una canaleta en espiral
}

CIVIL ENGINEERING

\author{
Effect of the secondary flow in the separation \\ of heavy metals in a spiral trough \\ Hernán J. Gómez Zambrano ${ }^{8 *}$ \\ *Departamento de Ingeniería Civil. Universidad de Nariño, Pasto, Colombia \\ shgomez@udenar.edu.co
}

(Recibido: Diciembre 19 de 2010 - Aceptado: Noviembre 16 de 2011)

\begin{abstract}
Resumen
En el presente artículo se reportan los resultados de la investigación experimental llevada a cabo para evaluar el efecto del flujo secundario en la separación de plomo contenido en una mezcla de agua y sedimentos, usando una canaleta en espiral con sección transversal del tipo FLG con radio interno de $0.025 \mathrm{~m}$ y radio externo de $0.15 \mathrm{~m}$ y un paso de espiral de $0.185 \mathrm{~m}$. Para establecer el procedimiento experimental se recurrió al método del análisis dimensional y al diseño de experimentos factorial aleatorizado, obteniéndose con dicho diseño un total de 16 corridas experimentales. Las variables de respuesta estudiadas fueron la concentración en peso de plomo en la descarga, la partición de agua, la remoción de sedimentos y la remoción de plomo, las cuales dependen de parámetros adimensionales como el número de Dean, la relación de radios y el coeficiente de arrastre promedio de las partículas sólidas que incluye el efecto de la granulometría. Con los datos experimentales se obtuvieron ecuaciones empíricas por medio de regresión lineal múltiple para cada una de las variables de respuesta.
\end{abstract}

Palabras Claves: Canaleta en espiral, Mezclas, Separación, Plomo, Metal Pesado.

\begin{abstract}
This paper reports the results of the experimental investigation carried out to evaluate the effect of the secondary flow in the separation of lead content in a mixture of water and sediments, using a spiral trough of FLG cross section, with internal radio of $0.025 \mathrm{~m}$ and an external radio of $0.15 \mathrm{~m}$ and a pitch of $0.185 \mathrm{~m}$. To establish the experimental procedure it was resorted to the method of the dimensional analysis and the randomized factorial design of experiments, being obtained with this design a total of 16 experimental data. The studied answer variables were the concentration of lead in the unload, the partition of water, the removal of sediments and the removal of lead, which depend of parameters non dimensional like Dean's number, relation of radios and the average drag coefficient that it includes the effect of the particles size. With the experimental data the author has obtained empiric equations by means of multiple lineal regression for each one of the responses variables.
\end{abstract}

Keywords: Spiral trough, Mixtures, Separation, Lead, Heavy metal. 


\section{Introducción}

En este artículo se presenta los resultados de la investigación denominada "Determinación del efecto del flujo secundario en la eficiencia de separación de metales pesados de una mezcla de material aluvial y agua", donde el flujo secundario fue logrado usando una canaleta en espiral con flujo a superficie libre. La mezcla de sólidos se preparó con plomo como metal pesado y sedimentos.

Para establecer los parámetros adimensionales a estudiar se utilizó el método del análisis dimensional y para conocer el número de ensayos experimentales y el orden de ejecución de los tratamientos experimentales, se realizó un diseño experimental con arreglo factorial completamente aleatorizado. Para la medición experimental se construyó un montaje de laboratorio el cual permitió recircular la mezcla de sedimento, plomo y agua; a través de la canaleta en espiral.

A pesar de que el flujo a través de una canaleta en espiral ha sido estudiado por varios investigadores, no se reporta una metodología de diseño que se pueda usar en la separación de metales como el plomo contenido dentro de una matriz de sedimentos. Los autores que han estudiado el fenómeno de flujo a través de una canaleta en espiral se encuentran Hollant-Batt (1995) quien expone algunas consideraciones prácticas que se deben tener en cuenta para el diseño de separadores en espiral, enfocando su estudio a los accesorios de la canaleta en espiral para mejorar la eficiencia de separación. Por otro lado Holland-Batt \& Holtham (1991), presentan dos tipos de ecuaciones para predecir la velocidad media del flujo, una usando el concepto de la ecuación de Manning y la otra el concepto de distribución logarítmica de velocidades, para la primera divide el flujo en zona interna, en zona de transición y zona externa de flujo principal, proponiendo ecuaciones para la velocidad en cada región de flujo. Das et al. (2007), comentan que el rendimiento de la espiral depende de los parámetros de diseño, como el diámetro, la altura, el número de espiras, el paso de la espira, la pendiente y la sección transversal de la canaleta; proponiendo una ecuación para estimar la velocidad media del flujo en una espiral en función de la geometría y las propiedades de las partículas; además presentan una metodología con base al balance de fuerzas que actúan sobre una partícula, para evaluar la distribución de partículas en sentido radial de la canaleta en espiral.

El flujo al pasar a través de una canaleta en espiral sufre alteración en la dirección, deformando las líneas de corriente del flujo, forzándolas a seguir trayectorias en forma de espiral desde la parte externa de la curva hacia a la parte interna. Dicho comportamiento del flujo en una canaleta en espiral, hace que se formen corrientes transversales adicionales a la línea de flujo principal, generando el flujo secundario. Estas corrientes son capaces de transportar las partículas sólidas más pesadas inmersas en el agua, desde la parte externa de la curva a la parte interna de la curva junto al fondo de la canaleta.

Para mayor comprensión del fenómeno del flujo secundario se incluye la Figura 1, en la cual se indica la dirección del flujo primario y flujo secundario y la dirección de la fuerza centrífuga. La distribución de las partículas en la descarga y el rebose dependen del valor de la fuerza centrífuga, si es mayor que la fuerza de arrastre producida por el flujo secundario las partículas más gruesas salen por el rebose, de lo contrario las partículas más gruesas salen por la descarga. En canaletas en espiral se ha observado que prima la segunda condición.

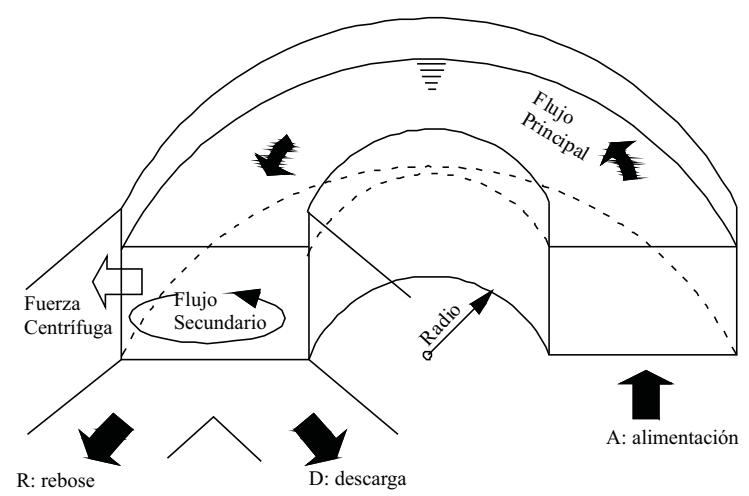

Figura 1. Esquema sobre flujo principal y flujo secundario en un conducto curvo. 
Para cuantificar el flujo secundario se utilizó el número de Dean de la mezcla $D e_{\mathrm{m}}$, el cual relaciona la fuerza de viscosidad que actúa sobre un fluido que se mueve en un conducto curvado y la fuerza centrífuga. Tomando como base la definición del número de Dean dada por Ookawara et al. (2004), el Autor propone un número de Dean para la canaleta en espiral de sección no circular con flujo de una mezcla.

Como resultado de la presente investigación se obtuvieron ecuaciones que relacionan las concentraciones de metal pesado, la partición de agua, la remoción de sedimentos y la remoción de metal pesado, con las variables que identifican las propiedades del agua, las de los sedimentos, las de la mezcla y la geometría de la canaleta en espiral. Con dichas correlaciones es posible saber cuál es la concentración de metal pesado a la salida de la canaleta, con relación a las condiciones de la entrada.

\section{Metodología}

\subsection{Montaje de laboratorio}

Para la realización de la investigación experimental se construyó un montaje de laboratorio como se indica esquemáticamente en la Figura 2 (a), el cual tiene los siguientes componentes principales: 1) tanque de succión de agua, 2) tolva de abastecimiento sólido, 3) bomba para impulsión de lodos, 4) tanque de cabeza constante para garantizar flujo uniforme, 5) mecanismo localizado antes del sedimentador para instalar la canaleta en espiral y 6) tanque desarenador para recuperar el sedimento y medir el caudal líquido a la salida del tanque por medio de vertederos triangulares. Para un mejor entendimiento de cómo fue la instalación se incluye la Figura 2 (b), en la cual se muestra el montaje construido.

a

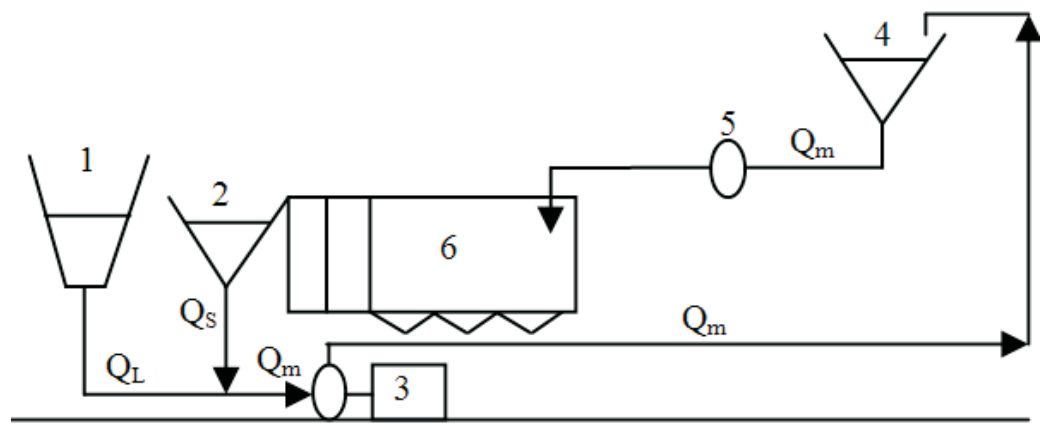

b

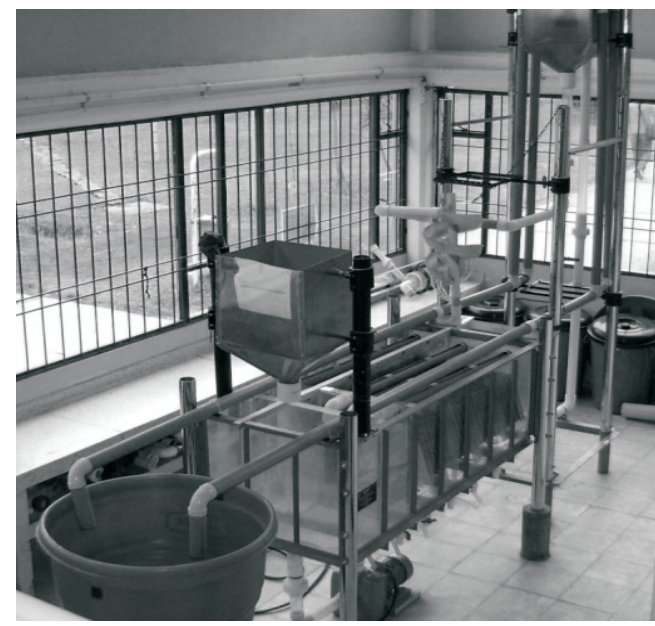

Figura 2. (a) Esquema general del montaje de laboratorio. (b)Fotografía del montaje construido en laboratorio. 
La canaleta en espiral ensayada tiene un radio interno de $0.025 \mathrm{~m}$, un radio externo de $0.15 \mathrm{~m}$, un paso de cada espira de $p=0.185 \mathrm{~m}$. La sección transversal seleccionada fue del tipo FLG (Fine Low Grade) usada para concentrar pulpas compuestas de sólidos finos con bajo contenido de metal pesado menor al 5\% en peso con base a la sección presentada por Holland-Batt \& Holtham (1991), construida a una escala de 1:2. En la Figura 3 (a) se indica la geometría vertical de la canaleta y en la Figura 3 (b) se indica la canaleta en espiral construida e instalada en laboratorio. La canaleta se moldeo en fibra de vidrio, sobre un molde de cemento, en secciones de $120^{\circ}$, las cuales se unieron alrededor del tubo central en PVC.

El sistema de descarga para la captación del metal pesado se diseñó usando orificios circulares de diámetro $d_{o}$ colocados en el fondo de la canaleta y conectados a mangueras de recolección, a diferentes radios sobre la sección transversal.

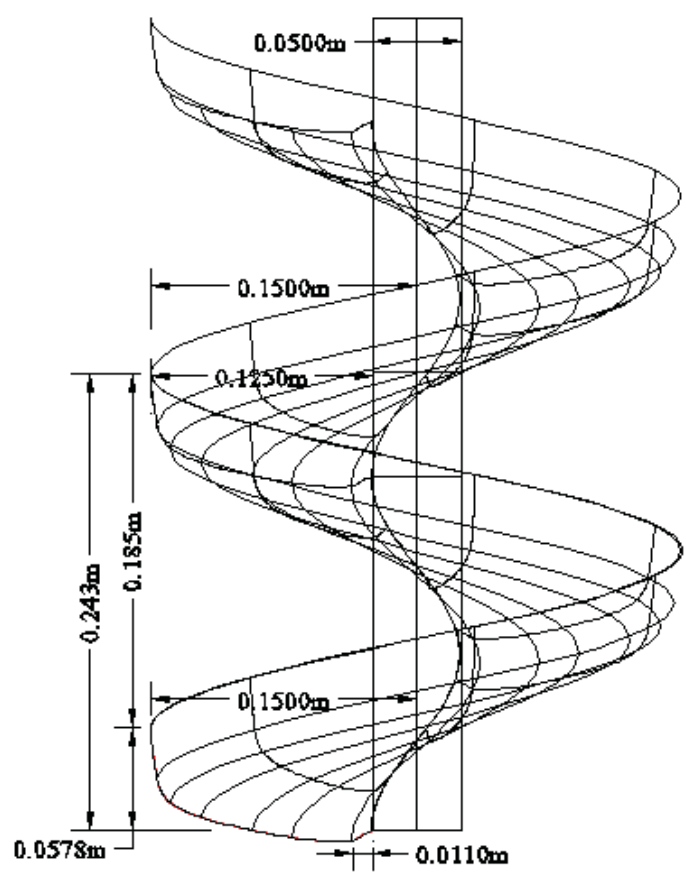

\subsection{Propiedades de los sólidos}

Las propiedades que se analizaron para el material sólido fueron la granulometría, la gravedad específica y el coeficiente de arrastre promedio.

La granulometría se realizó a la mezcla de sedimento y plomo, los sedimentos estuvieron comprendidos entre el tamiz 200 hasta el tamiz 10 y el plomo pulverizado entre el tamiz 200 y el 40. Se empleó tres tipos de sedimentos con diferente granulometría, clasificadas como $M_{1}, M_{2}$ y $M_{3}$, la muestra $M_{l}$ corresponde a arena proveniente de arenisca, la muestra $M_{2}$ extraída de lecho de río de origen volcánico de color café oscuro y la muestra $M_{3}$ corresponde a arena de río de origen volcánico de color gris. Debido a que el material sólido fue reutilizado y por desperdicios no controlables la granulometría cambió entre un ensayo y otro, se realizó la granulometría al material recuperado por la parte inferior o descarga y por la parte externa o rebose de la canaleta en espiral para cada

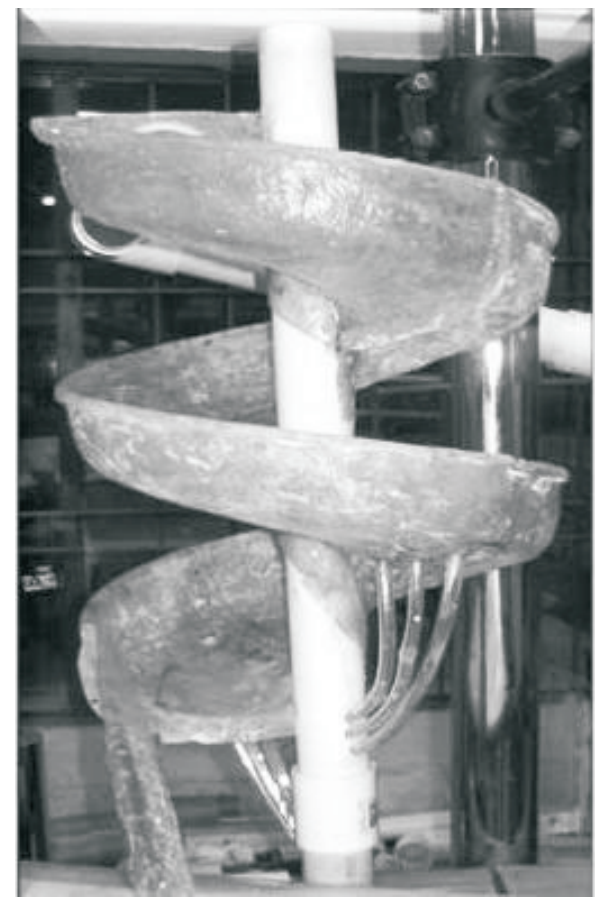

Figura 3. Canaleta en espiral. (a) Geometría de la canaleta. (b) Fotografía de la canaleta en espiral instalada en el montaje. 
ensayo y de dichas granulometrías se infirió la granulometría en la alimentación.

La gravedad específica se midió en laboratorio para cada tipo de muestra de sedimento y de plomo, donde, la gravedad específica para el sedimento de la muestra $M_{1}$ fue de $G_{s s}=2.627$; para la muestra $M_{2}$ fue de $G_{s s}=2.529$ y para $M_{3}$ fue de $G_{s s}$ $=2.399$. La gravedad específica del plomo medida fue de $G_{s P b}=11.302$.

\subsection{Definición de los factores independientes}

Los factores independientes se obtuvieron aplicando análisis dimensional a las variables involucradas en el fenómeno estudiado, en este sentido se obtuvo el Número de Dean $D \mathrm{e}_{\mathrm{m}}$, la relación de radios $R R$ y el coeficiente de arrastre promedio $C_{\mathrm{Dp}}$. La sección transversal de la canaleta y la representación geométrica de los símbolos de las variables usados en las ecuaciones de estos factores se indican en la Figura 4. A continuación se describe cada uno de los factores.

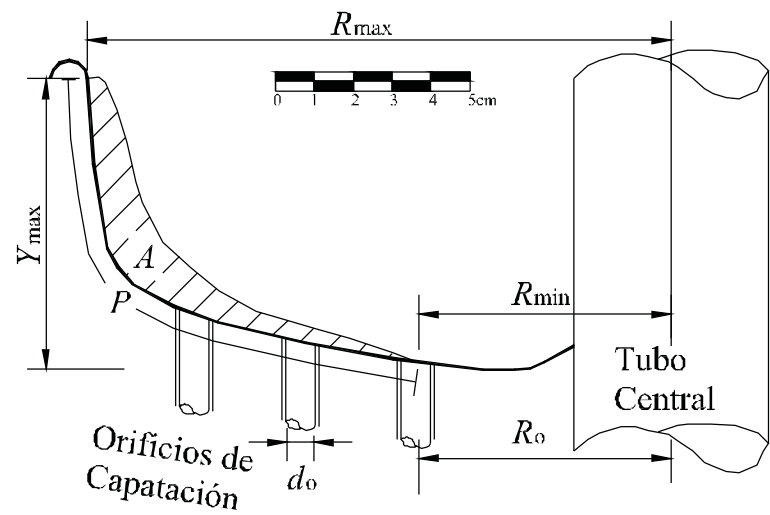

Figura 4. Esquema de la sección transversal de la canaleta en espiral FLG (Fine Low Grade), usada para concentrar pulpas con sólidos finos de bajo contenido de metal pesado.

Número de Dean. Modificando la ecuación dada por Ookawara et al. (2004) para este parámetro y adaptándola al flujo sobre una canaleta en espiral, se tiene:

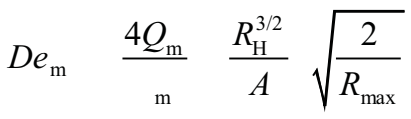

Donde, $\mathrm{R}_{\max }$ es el radio máximo que alcanza el agua en la canaleta en metros con un valor de $0.150 \mathrm{~m}, R_{\mathrm{H}}$ es el radio hidráulico de la sección transversal en $(\mathrm{m})$, calculado como área sobre perímetro $R_{\mathrm{H}}=A / P, P$ es el perímetro mojado en (m), tomado como la distancia medida en la sección transversal de la canaleta en contacto con el agua, $A$ es el área mojada de la sección transversal en metros cuadrados $\left(\mathrm{m}^{2}\right)$, calculada como $A=Q_{\mathrm{m}} / V_{\mathrm{m}}$, donde $\mathrm{V}_{\mathrm{m}}$ es la velocidad superficial del flujo de la mezcla en metros por segundo $(\mathrm{m} / \mathrm{s})$, medida con un flotador y calculada como $V_{\mathrm{m}}=L / t$, donde $L$ es la longitud de la canaleta para un radio promedio en (m) y $t$ es el tiempo de recorrido del flotador entre la entrada y la salida de la canaleta en segundos (s). $Q_{\mathrm{m}}$ es el caudal de la mezcla en $\left(\mathrm{m}^{3} / \mathrm{s}\right)$, dado como: $Q_{\mathrm{m}}=Q_{\mathrm{L}}+Q_{\mathrm{s}}+Q_{\mathrm{pb}}$, donde, $Q_{\mathrm{bp}}$ es el caudal de plomo en $\left(\mathrm{m}^{3} / \mathrm{s}\right), Q_{\mathrm{s}}$ es el caudal de sedimentos en $\left(\mathrm{m}^{3} / \mathrm{s}\right)$ y $Q_{\mathrm{L}}$ es el caudal de agua limpia o caudal líquido en $\left(\mathrm{m}^{3} / \mathrm{s}\right)$, este último se adoptó como variable manipulable en laboratorio para hacer variar el número de Dean por su facilidad de manipulación. $v_{\mathrm{m}}$ es la viscosidad cinemática de la mezcla en $\left(\mathrm{m}^{2} / \mathrm{s}\right)$, calculada según la metodología dada por Cheng (1997b). Para el cálculo de $V_{\mathrm{m}}$ se utilizó los datos experimentales para calibrar la Ec. (2), dada por Holland-BattA., B. (1989).

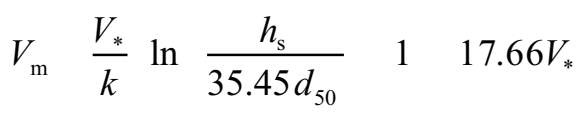

Donde, $h_{\mathrm{s}}$ es la profundidad de la mezcla en la canaleta en (m), con los datos experimentales se obtuvo un valor de $h_{\mathrm{s}}=Y_{\text {max }} / 14$, siendo $Y_{\max }$ la altura máxima del agua en la canaleta en (m) correspondiente a $R_{\max }, d_{50}$ es el diámetro en $(\mathrm{m})$ de la abertura de la malla del tamiz por el cual pasa el $50 \%$ del sedimento, $k$ es la constante de Von Karman igual a 0.3 para flujo de suspensiones; $V_{*}$ es la velocidad de corte en $(\mathrm{m} / \mathrm{s})$, calculada con Ec. (3).

$$
V_{*} v \sqrt{g h_{\mathrm{s}} \operatorname{sen}(v)}
$$


Donde, $g$ es la aceleración de la gravedad en $\left(\mathrm{m} / \mathrm{s}^{2}\right), v$ es el ángulo longitudinal de la canaleta calculado con Ec. (4)

$$
v v \tan ^{v 1} \frac{p}{v 2 v R_{\max }} v
$$

Donde, $p$ es el paso de la espiral igual a $0.185 \mathrm{~m}$.

Relación de radios. Relaciona el radio de localización de los orificios de drenaje o captación de metal pesado $R_{o}$ y el radio máximo de la canaleta $R_{\max }$. $R R$ se calculó con la Ec. (5).

$$
R R \vee \frac{R_{\mathrm{o}}}{R_{\max }}
$$

Para garantizar tres variaciones de la relación de radios $R R$ se seleccionó el radio $R_{0}$ como variable manipulable en laboratorio, el cual tomó los siguientes valores $0.0656 \mathrm{~m}, 0.0952 \mathrm{~m}$ y $0.1225 \mathrm{~m}$, medidos desde el centro del eje del tubo soporte. El diámetro de los orificios de captación $d_{\mathrm{o}}$ fue de 7.5 milímetros.

Coeficiente de arrastre promedio de las particulas $\left(C_{\mathrm{Dp}}\right)$. Con la granulometría y la gravedad específica se calculó el coeficiente de arrastre de las partículas $C_{\mathrm{D}}$, el cual relaciona las propiedades de los sedimentos y del fluido, calculado con la metodología dada por Cheng (1997a), junto con el procedimiento de Condolios nombrado por Graf (1984). Debido a que los sedimentos y plomo usados no son de granulometría uniforme, se calculó para cada rango de tamaños el valor de $C_{\mathrm{D}}$ con la metodología de Cheng (1997a) y luego se calculó un valor ponderado de acuerdo al porcentaje de sedimento dentro del rango, aplicando la ecuación de Condolios, dada por Ec. (6):

$$
\begin{aligned}
& \sqrt{C_{\mathrm{Dp}}} \vee \underset{\mathrm{i}+1}{\vee} P_{\mathrm{i}} \sqrt{C_{\mathrm{Di}}} \vee P_{1} \sqrt{C_{\mathrm{D} 1}} \vee \\
& P_{2} \sqrt{C_{\mathrm{D} 2}} \vee P_{3} \sqrt{C_{\mathrm{D} 3}} \vee \ldots \vee P_{\mathrm{nJ}} \sqrt{C_{\mathrm{DJ}}}
\end{aligned}
$$

Donde, $J$ es el número de rangos entre tamices; $P_{1}$, $P_{2}, \ldots, P_{\mathrm{J}}$, son los porcentajes en peso del sólido correspondientes a dos tamices consecutivos tomados de la granulometría de cada muestra y $C_{\mathrm{D} 1}, C_{\mathrm{D} 2}, \ldots, C_{\mathrm{DJ}}$, son los coeficientes de arrastre de cada rango de tamaños, tomando para su cálculo el diámetro promedio del rango $d_{\mathrm{p}}$ dado como $d_{\mathrm{p}}=$ $\left(d_{\mathrm{i}}+d_{\mathrm{s}}\right) / 2, d_{\mathrm{i}}$ es el diámetro inferior del rango en $(\mathrm{m})$ y $d_{\mathrm{s}}$ es el diámetro superior del mismo en (m). El coeficiente de arrastre promedio $C_{\mathrm{Dp}}$ corresponde al valor promedio de la mezcla de sedimento y plomo, debido a que en la práctica es difícil conocer este valor por separado. En la metodología dada por Cheng (1997a) para calcular el coeficiente de arrastre $C_{\mathrm{Dp}}$, se modificó el parámetro adimensional de densidades $v$ dado como $v=\left(\nu_{\mathrm{M}}-\nu_{\mathrm{w}}\right) / \nu_{\mathrm{w}}$, donde $\nu_{\mathrm{w}}$ es el peso específico del agua en Newtons por metro cúbico $\left(\mathrm{N} / \mathrm{m}^{3}\right)$ a temperatura de ensayo, $\nu_{\mathrm{sM}}$ es el peso específico de los sólidos en $\left(\mathrm{N} / \mathrm{m}^{3}\right)$ el cual incluye los sedimentos y el metal pesado, calculado con Ec. (7):

$$
v_{\mathrm{sM}} \vee\left(1 \vee C_{\mathrm{VM}}\right) v_{s} \vee C_{\mathrm{VM}_{\mathrm{M}}} v_{\mathrm{M}}
$$

Donde, $C_{\mathrm{VM}}$ es la concentración volumétrica de metal pesado dentro de la mezcla de sedimento y metal, $v_{\mathrm{M}}$ es el peso específico del metal en $\left(\mathrm{N} / \mathrm{m}^{3}\right)$ y $v_{\mathrm{s}}$ es el peso específico de los sedimentos en $\left(\mathrm{N} / \mathrm{m}^{3}\right)$.

\subsection{Definición de las variables de respuesta}

La variables de respuesta seleccionadas y que son de interés en el fenómeno estudiado son la partición de agua $P A$, la remoción de sedimentos $R_{\mathrm{s}}$, la remoción de plomo $R_{\mathrm{Pb}}$ y la concentración de metal pesado en peso por la descarga $C_{\mathrm{PbD}}$. A continuación se realiza una explicación de cada una de estas variables.

Partición de agua. Esta variable se incluye, debido a que es importante conocer la distribución del agua a la salida del separador tanto en el rebose como en la descarga, con el fin de conocer el grado de espesamiento y ser usada en etapas posteriores de separación. La partición de agua está definida 
como la relación de caudales de agua, entre la descarga de la canaleta en espiral $Q_{\mathrm{D}}$ y el caudal de agua total en la alimentación de la canaleta en espiral $Q_{\mathrm{A}}$, expresada en Ec. (8):

$$
P_{\mathrm{A}} \vee \frac{Q_{\mathrm{D}}}{Q_{\mathrm{A}}}
$$

Remoción de sedimentos. Definida como la relación entre el peso de sedimentos de la descarga $W_{\text {sD }}$ y el peso total de sedimentos en la alimentación $W_{\mathrm{sA}}$, de la canaleta en espiral, dada en Ec. (9):

$$
R_{\mathrm{s}} v \frac{W_{\mathrm{sD}}}{W_{\mathrm{sA}}}
$$

Remoción de plomo. Definida como la relación de peso de plomo entre la descarga $W_{\mathrm{PbD}}$ y la alimentación $W_{\mathrm{PbA}}$, de la canaleta en espiral, expresada en Ec. (10):

$$
R_{\mathrm{Pb}} \vee \frac{W_{\mathrm{PbD}}}{W_{\mathrm{PbA}}}
$$

Concentraciones de plomo en la descarga. Definida como la relación del peso de plomo recuperado por la descarga $W_{\mathrm{PbD}}$ y el peso total de plomo mas sedimento recuperado por la misma descarga $W_{\mathrm{sPbD}}$, expresada en Ec. (11):

$$
C_{P b D} \vee \frac{W_{P b D}}{W_{S P b D}}
$$

Introduciendo Ec. (9) y Ec. (10) en Ec.(11) se obtiene la Ec. (12) para $C_{\mathrm{PbD}}$.

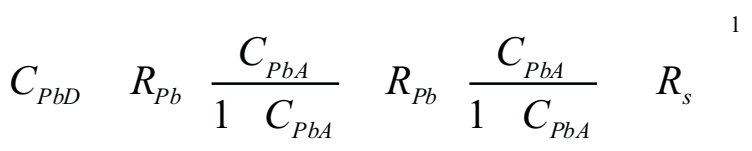

Donde, $C_{\mathrm{PbA}}$ es la concentración de plomo en la alimentación, valor que debe ser conocido $\mathrm{y}$ relaciona el peso de plomo en la alimentación $W_{\mathrm{PbA}}$ y el peso de sedimento y plomo en la alimentación $W_{\mathrm{sPbA}}$, por lo tanto se obtiene que $C_{\mathrm{PbA}}=$ $W_{\mathrm{PbA}} / W_{\mathrm{sPbA}}$.

\subsection{Diseño experimental}

Para el diseño experimental se usó la metodología dada por Gutiérrez \& De la Vara (2008), optándose por un diseño factorial mezclado con cuatro puntos al centro $\left(3 * 2^{3-1}+4\right)$ completamente aleatorizado, para un total de 16 tratamientos. El arreglo experimental se realizó con un software estadístico especializado.

De esta forma fue posible obtener una relación funcional adimensional de parámetros independientes que influyen en cada una de las variables de respuesta representadas por $v_{\mathrm{C}}$ como se indica en Ec. (13).

$$
v_{\mathrm{C}} \vee f \mathrm{~W} e_{\mathrm{m}}, R R, C_{\mathrm{Dp}} \mathrm{V}
$$

\subsection{Procedimiento de medición}

Medición de datos en el montaje. Para minimizar los errores de medición se procedió a grabar en video el sistema de medición del montaje de laboratorio para cada corrida experimental y luego se obtuvieron los valores del video. Para cada experimento se procedió de la siguiente manera:

- Garantizar un flujo permanente de agua a través de la canaleta en espiral.

- Suministrar el caudal sólido, por medio de una tolva con orificios calibrados.

- Grabar con cámara de video los dispositivos de medición en el tiempo de duración del ensayo.

- Recuperar la mezcla de sedimento y plomo al final de cada ensayo en cada salida de la canaleta en espiral, secar por 24 horas a $120^{\circ} \mathrm{C}$ y pesar los sólidos por separado.

- Realizar las granulometrías a las muestras recuperadas correspondientes al paso anterior, de forma separada correspondiente a cada salida.

- Medir la concentración de plomo de las muestras recuperadas por cada salida.

Las cantidades físicas medidas mediante el uso de video, fueron las siguientes: altura del vertedero correspondiente a la descarga de la canaleta en espiral $h_{\mathrm{vD}}$; atura del vertedero correspondiente a la salida externa del canaleta o rebose $h_{\mathrm{vR}}$; temperatura en grados centígrados $T$ registrada con un termómetro capilar digital; tipo de muestra de sedimentos $\left(M_{1}, M_{2}, M_{3}\right)$ y el tipo de captación de metal pesado. 
Medición del caudal líquido. El caudal del agua se calculó con tres vertederos triangulares localizados a la salida de los sedimentadores, donde los vertederos 1 y 2 miden el caudal líquido de la salida externa o rebose de la canaleta en espiral y el vertedero 3 mide el caudal a la salida interna o descarga de la canaleta en espiral. La ecuación de calibración para los vertederos 1-2 es $Q_{\mathrm{R}}=1.2424086\left(h_{\mathrm{vR}}\right)^{2.4113}$ y la ecuación de calibración del vertedero 3 es $Q_{\mathrm{D}}=0.6472880\left(h_{\mathrm{vD}}\right)^{2.4143}$; donde, $h_{\mathrm{vR}}$ y $h_{\mathrm{vD}}$ en $(\mathrm{m})$ y $Q$ en $\left(\mathrm{m}^{3} / \mathrm{s}\right)$.

Medición de la concentración de plomo. La concentración de plomo en los sedimentos se midió utilizando dos métodos. Para concentraciones bajas menores al 10\% se utilizó los datos obtenidos por el método de digestión ácida HN03-HCLO4-HC1 con técnica de Espectrofotometría de Absorción Atómica realizado por el Laboratorio Especializado de la Universidad de Nariño, y para concentraciones mayores al $10 \%$ se utilizó los datos obtenidos por el método basado en el principio de Arquímedes. Las concentraciones medidas correspondieron al rebose y la descarga, a partir de las cuales, se calculó la concentración en la alimentación. El método basado en el principio de Arquímedes no es muy preciso para concentraciones menores al $10 \%$ debido a la precisión de la balanza utilizada de \pm 0.01 gramos, la cual genera errores altos en el método para concentraciones bajas de metal. El método de Espectrofotometría no es muy aplicable a concentraciones altas de metal, debido a que el plomo se precipita produciendo inexactitudes en los datos. Para muestras con concentraciones muy altas también se utilizó el método de Digestión Acida HF.

En el laboratorio se midió las concentraciones de plomo de las muestras de la descarga y rebose de la canaleta y por balance de masas se calculó la concentración en la alimentación. Para seleccionar las muestras que fueron enviadas al laboratorio, se utilizó el método de cuarteo, tomando un peso de diez gramos por muestra. Para cada ensayo se obtuvieron dos muestras, para un total de 32 muestras analizadas.

Datos de laboratorio. En la Tabla 1, se indican los valores de los datos tomados en laboratorio, donde $N^{o}$ indica el número de datos, $C_{\mathrm{Dp}}$ es el coeficiente de arrastre promedio en la alimentación, $R R$ es la relación de radios, $A$ es el área mojada en

Tabla 1. Datos de laboratorio para mezcla de agua, sedimento y plomo.

\begin{tabular}{|c|c|c|c|c|c|c|c|c|c|c|c|c|c|}
\hline$N^{\circ}$ & $C_{\mathrm{Dp}}$ & $R R$ & $\begin{array}{c}A, \\
\mathrm{~cm}^{2}\end{array}$ & $\begin{array}{c}\boldsymbol{P}, \\
\mathrm{mm}\end{array}$ & $\begin{array}{c}\boldsymbol{W}_{\text {SMD }}, \\
\mathrm{N}\end{array}$ & $\begin{array}{c}\boldsymbol{W}_{\text {SMR, }} \\
\mathrm{N}\end{array}$ & $\begin{array}{c}\boldsymbol{h}_{\mathrm{VR},} \\
\mathrm{mm}\end{array}$ & $\begin{array}{c}\boldsymbol{h}_{\mathrm{VD},} \\
\mathrm{mm}\end{array}$ & $\begin{array}{c}T, \\
{ }^{\circ} \mathrm{C}\end{array}$ & $\begin{array}{c}\boldsymbol{Q}_{\mathrm{Pb}}, \\
\mathrm{cm}^{3} / \mathrm{s}\end{array}$ & $\begin{array}{c}Q_{\mathrm{s}}, \\
\mathrm{cm}^{3} / \mathrm{s}\end{array}$ & $\begin{array}{l}C_{\mathrm{PbA}}, \\
\%\end{array}$ & $\begin{array}{l}C_{\mathrm{PbD}}, \\
\%\end{array}$ \\
\hline 1 & 14.95 & 0.43 & 7.0 & 124 & 4.9 & 91.8 & 46 & 5 & 18.5 & 0.34 & 41.16 & 3.74 & 66.76 \\
\hline 2 & 3.48 & 0.81 & 4.4 & 129 & 24.7 & 72.9 & 36 & 14 & 19.9 & 0.46 & 37.72 & 4.95 & 16.25 \\
\hline 3 & 13.74 & 0.43 & 4.2 & 122 & 2.4 & 95.3 & 37 & 2 & 18.9 & 0.29 & 38.99 & 3.40 & 61.08 \\
\hline 4 & 7.91 & 0.63 & 4.3 & 121 & 10.8 & 86.7 & 37 & 4 & 21.2 & 0.69 & 36.76 & 7.69 & 29.33 \\
\hline 5 & 3.42 & 0.81 & 6.8 & 115 & 23.4 & 74.1 & 45 & 16 & 21.7 & 0.54 & 37.35 & 5.88 & 18.03 \\
\hline 6 & 4.19 & 0.44 & 1.6 & 94 & 7.8 & 90.0 & 20 & 2 & 18.5 & 0.32 & 36.98 & 3.59 & 28.28 \\
\hline 7 & 3.53 & 0.83 & 1.7 & 103 & 34.1 & 63.7 & 19 & 12 & 18.9 & 0.88 & 35.88 & 9.57 & 13.60 \\
\hline 8 & 8.41 & 0.63 & 4.3 & 120 & 15.8 & 81.6 & 37 & 4 & 21.9 & 0.57 & 37.72 & 6.31 & 29.33 \\
\hline 9 & 13.26 & 0.83 & 1.7 & 101 & 37.2 & 60.5 & 19 & 12 & 19.3 & 0.76 & 38.99 & 8.43 & 21.72 \\
\hline 10 & 11.83 & 0.81 & 6.7 & 113 & 21.3 & 76.1 & 45 & 15 & 21.5 & 0.68 & 39.16 & 7.60 & 31.96 \\
\hline 11 & 8.11 & 0.63 & 4.3 & 123 & 11.9 & 85.5 & 37 & 4 & 22.4 & 0.51 & 37.35 & 5.78 & 28.70 \\
\hline 12 & 14.64 & 0.44 & 1.7 & 105 & 6.8 & 90.9 & 20 & 3 & 19.6 & 0.46 & 38.49 & 5.31 & 51.80 \\
\hline 13 & 5.15 & 0.43 & 6.9 & 132 & 3.5 & 93.8 & 46 & 4 & 21.6 & 0.61 & 34.92 & 6.94 & 82.66 \\
\hline 14 & 7.62 & 0.63 & 4.2 & 124 & 14.2 & 83.2 & 37 & 4 & 22.3 & 0.73 & 37.03 & 8.05 & 28.77 \\
\hline 15 & 4.13 & 0.43 & 4.2 & 117 & 1.8 & 95.9 & 37 & 2 & 22.9 & 0.69 & 37.95 & 7.29 & 81.79 \\
\hline 16 & 11.60 & 0.81 & 4.1 & 115 & 23.7 & 74.0 & 36 & 14 & 22.5 & 0.52 & 37.93 & 6.12 & 23.39 \\
\hline
\end{tabular}


centímetros cuadrados, $P$ es el perímetro mojado en milímetros, $W_{\text {sMD }}$ es el peso de arena y plomo recuperado en la descarga en Newtons $(\mathrm{N}), W_{\text {sMR }}$ es el peso de arena y plomo recuperado en el rebose en $(\mathrm{N}), h_{\mathrm{vR}}$ es la altura del agua sobre el vertedero triangular que mide el caudal en el rebose en milímetros, $h_{\mathrm{vD}}$ es la altura del agua sobre el vertedero triangular que mide el caudal en la descarga en milímetros, $\mathrm{T}$ es la temperatura del agua en grados centígrados $\left({ }^{\circ} \mathrm{C}\right), Q_{\mathrm{pb}}$ es el caudal volumétrico de plomo en centímetros cúbicos por segundo $\left(\mathrm{m}^{3} / \mathrm{s}\right), Q_{\mathrm{s}}$ es el caudal volumétrico de sedimentos en $\left(\mathrm{cm}^{3} / \mathrm{s}\right), C_{\mathrm{pbA}}$ es la concentración en peso y porcentaje de plomo contenido en la mezcla en la alimentación y $C_{\mathrm{pbD}}$ es la concentración en peso y porcentaje de plomo contenido en la mezcla en la descarga. En los cálculos las unidades usadas fueron las correspondientes al Sistema Internacional.

\section{Resultados y discusión}

A partir de los datos experimentales, se obtuvieron nuevas ecuaciones empíricas para la partición de agua, la remoción de sedimentos y la remoción de metal pesado, aplicando regresión lineal múltiple (RLM), se obtuvo la Ec. (14), Ec. (15) y Ec.(16), como se indica a continuación:

\section{Partición de agua.}

$$
P A \vee 10^{0.408054} D e_{\mathrm{m}}{ }^{{ }^{0.488216}} R R^{5.9528} C_{\mathrm{Dp}}{ }^{0.24548}
$$

Remoción de plomo.

$$
R_{\mathrm{Pb}} \vee 10^{\vee 0.307632} D e_{\mathrm{m}}^{\nu 0.0111963} R R^{0.851477} C_{\mathrm{Dp}}{ }^{0.3610049}
$$

Remoción de sedimento.

$$
R_{\mathrm{s}} \vee 10^{0.705863} D e_{\mathrm{m}}^{v 0.359818} R R^{4.60447} C_{\mathrm{Dp}}^{0.259964}
$$

Altura máxima del agua en la canaleta $\left(Y_{\max }\right)$.

$$
\frac{Y_{\max }}{R_{\max }} \vee 10^{\mathrm{v} 0.99631} D e_{\mathrm{m}}^{0.181165} R R^{\mathrm{v} 0.104983} C_{\mathrm{Dp}}{ }^{v 0.0122973}
$$

Donde, $Y_{\max }$ se mide desde el punto más bajo de la sección transversal de la canaleta. Los valores del análisis estadístico para las Ec. (14), (15), (16) y (17), se indican en su orden, p-valor: (0.0001, $0.0403,0.0000,0.0004)$ y $r$ cuadrado $\left(r^{2}\right)$ en porcentaje: $(81.91,48.61,87.80,77.00)$, respectivamente.

La concentración de plomo en la descarga $C_{\mathrm{PbD}}$ se calculó remplazando la Ec. (15) y Ec. (16) en la Ec. (12).

\subsection{Verificación de las correlaciones}

Para evaluar el error de predicción de la Ec. (12), se utilizó el criterio de error promedio $\bar{E}$, el cual es dado en Ec. (18):

$$
\bar{E}=\frac{{ }_{\mathrm{i} v 1}^{\mathrm{ND}} v E_{\mathrm{i}} \vee}{N D}
$$

Donde $N D$ es el número de experimentos, $E_{\mathrm{i}}$ es el error de predicción para cada uno de los datos en $\%$, calculado con Ec. (19):

$$
E_{\mathrm{i}}=\left|\frac{\text { med-cal }}{\text { med }}\right| 100
$$

Donde, med es el valor medido y cal es el valor calculado, correspondiente a $C_{\mathrm{PbD}}$. Aplicando Ec. (18) se obtuvo un error promedio de $\bar{E}=20.15 \%$ para Ec.(12). En la Figura 5, se indica la representación gráfica de los valores medidos y calculados con la Ec. (12).

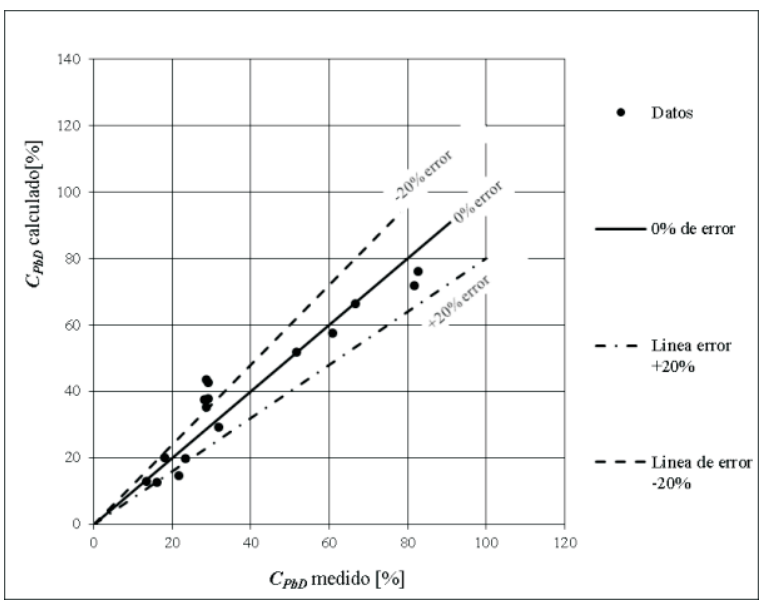

Figura 5. Valores medidos y calculados con Ec. (12). 


\section{Conclusiones}

Se establecen nuevas ecuaciones empíricas para predecir el grado de enriquecimiento del concentrado plomo a través de una canaleta en espiral, con un buen grado de exactitud, obteniéndose la Ec. (12) con base al balance de masa entre la entrada y la salida de la canaleta en espiral y calibrada con datos experimentales. Esta ecuación relaciona las propiedades geométricas de la canaleta en espiral con las propiedades de los sedimentos, las propiedades del metal pesado y las propiedades del flujo de la mezcla.

Se demuestra que el flujo secundario generado por la curvatura del flujo principal, puede ser utilizado en la separación de metales pesados contenidos en una matriz de material aluvial más liviano.

El procedimiento experimental aplicado en la investigación, puede ser utilizado para el estudio de otro tipo de canaletas en espiral. Esto debido a que los resultados obtenidos son válidos solamente para la geometría de la espiral presentada en este artículo.

\section{Agradecimientos}

El autor expresa los más sinceros agradecimientos a los estudiantes David Pabón, Manuel Perenguez y Andrés Ortiz, quienes trabajaron con gran responsabilidad en la ejecución de la investigación y a Roberto García encargado del laboratorio de hidráulica por su desinteresada colaboración.

\section{Referencias bibliográficas}

Cheng, N., S. (1997a). Simplified settling velocity formula for sediment particle. Journal of Hydraulic Engineering 123 (2), 149-152.

Cheng, N.-S., (1997b). Effect of concentration on settling velocity of sediment particles. Journal of Hydraulic Engineering 123 (8), 728-731.

Das, S., K., Godiwalla, K.M., Panda, L., Bhattacharya, K.K., Singh, R., Mecrotra, S.P. (2007). Mathematical modeling of separation characteristics of a coal-washing spiral. Int. J. Miner. Process. 84, 118132.
Graf, H. W., (1984). Hydraulics of sediment transport. New York: McGraw.Hill, Inc.

Gutiérrez, P. H., De La Vara, S. R. (2008). Análisis $y$ diseño de experimentos. México D.F: McGrawHill, Hill.

Holland-Batt A., B. (1989). Spiral Separation: theory and simulation. Trans. Instn. Min. Metall. (Sect. C: Mineral Process. 98 (January-April), C46-C60.

Holland-Batt, Holtham, P., N. (1991). Particle and fluid motion on spiral separators. Minerals Engineering. 4(3-4), 457-482.

Holland-Batt A., B. (1995). The dynamics of sluice and spiral separations. Minerals Engineering. 8(1-2), 3-21.

Ookawara, S., Higashi, R., Street, D., Ogawa, K. (2004). Feasibility study on concentration of slurry and classification of contained particles by microchannel. Chemichal Engineering Journal. 101, 171-178. 